\title{
Fiber Bragg grating-based shear strain sensors for adhesive bond monitoring
}

Sulejmani, Sanne, Sonnenfeld, Camille, Geernaert, Thomas, Van Hemelrijck, Danny, Luyckx, Geert, et al.

Sanne Sulejmani, Camille Sonnenfeld, Thomas Geernaert, Danny Van Hemelrijck, Geert Luyckx, Pawel Mergo, Waclaw Urbanczyk, Karima Chah, Christophe Caucheteur, Patrice Mégret, Hugo Thienpont, Francis Berghmans, "Fiber Bragg grating-based shear strain sensors for adhesive bond monitoring," Proc. SPIE 9128, Micro-structured and Specialty Optical Fibres III, 91280D (2 May 2014); doi: 10.1117/12.2050338

SPE. Event: SPIE Photonics Europe, 2014, Brussels, Belgium 


\title{
Fiber Bragg grating-based shear strain sensors for adhesive bond monitoring.
}

\author{
Sanne Sulejmani ${ }^{\mathrm{a}^{*}}$, Camille Sonnenfeld ${ }^{\mathrm{a}}$, Thomas Geernaert ${ }^{\mathrm{a}}$, Danny Van Hemelrijck ${ }^{\mathrm{b}}$, Geert \\ Luyckx ${ }^{\mathrm{c}}$, Pawel Mergo ${ }^{\mathrm{d}}$, Waclaw Urbanczyk ${ }^{\mathrm{e}}$, Karima Chah ${ }^{\mathrm{f}}$, Christophe Caucheteur ${ }^{\mathrm{f}}$, Patrice \\ Mégret $^{\mathrm{f}}$, Hugo Thienpont ${ }^{\mathrm{a}}$, Francis Berghmans ${ }^{\mathrm{a}}$ \\ ${ }^{a}$ Brussels Photonics Team, Vrije Universiteit Brussel, Pleinlaan 2, 1050 Brussel, Belgium; ${ }^{b}$ Dept. \\ of Mechanics of Materials and Constructions, Vrije Universiteit Brussel, Pleinlaan 2, 1050 \\ Brussel, Belgium; ${ }^{\mathrm{c}}$ Dept. of Materials Science and Engineering, Universiteit Gent, \\ Technologiepark 903, 9052 Gent, Belgium; 'Dept. of Optical Fiber Technology, Marie Curie- \\ Sklodowska University, Pl. M. Curie-Sklodowskiej 3, 20-031 Lublin, Poland; ' Institute of \\ Physics, Wroclaw University of Technology, Wybrzeze Wyspianskiego 27, 50-370 Wroclaw,

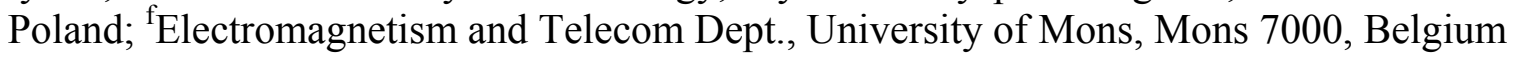

\begin{abstract}
The application of shear stress sensors in structural health monitoring remains limited because current sensors are either difficult to implement, they feature a low measurement resolution or the interrogation of the output signal is complex. We propose to use fiber Bragg grating-based sensors fabricated in dedicated highly birefringent microstructured optical fibers. When embedded in a host material, the orientation angle of the fiber should be chosen such that their polarization axes are aligned parallel with the direction of maximum shear stress when the host is mechanically loaded. We present experimental results of sensors embedded in the adhesive layer of single lap and double lap structural joints. These tests demonstrate that when the joints are tension loaded, the embedded sensors have a shear stress sensitivity of around $60 \mathrm{pm} / \mathrm{MPa}$. We study the influence of the adhesive material on the sensor response, as well as the influence of sensor orientation and location in the bond line. Finally, we demonstrate the minimal thermal cross-sensitivity of the shear stress sensitivity of this sensor.
\end{abstract}

Keywords: fiber Bragg grating, microstructured optical fiber, shear strain sensor, structural health monitoring, adhesive bond

\section{INTRODUCTION}

Fiber Bragg grating (FBG) based sensors offer well known advantages, including low weight, multiplexing capabilities, immunity to electromagnetic interference and the ability to be embedded in a non-intrusive manner in materials and structures. FBG sensors have become very popular for strain monitoring applications, in particular for structural health monitoring (SHM) purposes.

Axial strain sensing with FBG sensors written in standard single mode optical fibers has become a successful alternative for electrical strain gauges. In recent years, promising results have also been reported on FBG sensors fabricated in highly birefringent fibers for transverse strain sensing [1]. However, the main drawback of transverse strain sensing with conventional polarization maintaining fibers is their significant thermal cross-sensitivity and their rather low transverse strain sensing resolution. Microstructured optical fibers (MOFs) have been proposed to overcome these issues. High birefringence in these fibers is induced by an asymmetric air hole geometry and only a small and low GeO2-doped region is needed to allow for Bragg grating inscription using conventional UV inscription techniques. Moreover, their transverse strain sensitivity can be enhanced by properly tuning the air hole geometry, i.e. by altering the number of air holes, their sizes and/or their positions in the cross-section of the fiber. Results of the 'butterfly' microstructured optical fiber Bragg grating (MOFBG) sensor demonstrate that by designing dedicated MOFs one can improve the transverse strain sensitivity by an order of magnitude compared to conventional polarization maintaining fibers [2]. 
Besides the ability to sense the normal stress mentioned above, shear stress sensing is also important for SHM purposes. Shear stress monitoring is particularly interesting when dealing with adhesive bonds, since excessive shear stress could lead to disbonding. Continuously assessing shear stress could therefore help to prevent catastrophic failure of structures. Although one prefers adhesive bonding in many applications over other fastening techniques such as rivets, bolts or welding, no adequate technique exists at present to control the manufacturing quality of an adhesive bond or to monitor its quality during operation. Fiber optic shear stress sensing has received fairly little attention so far. We have shown recently that the butterfly MOFBG sensor mentioned above can also be used for shear stress sensing [3]. By aligning the optical axes parallel with the directions of principal stress in a shear loaded material, the butterfly MOFBG sensor will detect the shear load induced transverse stress.

In this paper we build further on our previous work and we describe how we embedded several butterfly MOFBG sensors in an adhesive bond to map the shear stress distribution. We placed the sensors at various locations along the overlap of single lap joints (SLJ) and double lap joints (DLJ), and we experimented with different types of adhesives. Because of the asymmetric air hole geometry of the butterfly MOF, angular misalignment will influence the shear stress sensitivity. We therefore experimentally investigated the influence of angular alignment when the sensor is embedded in a SLJ. These results demonstrate that adapting the angular orientation of the butterfly MOFBG sensor in a controlled manner can enable either shear stress or transverse stress sensing. Finally, we also touch upon the low cross-sensitivity of the butterfly MOFBG shear stress sensor to temperature.

\section{BRAGG GRATING-BASED SHEAR STRESS SENSING WITH HIGHLY BIREFRINGENT MICROSTRUCTURED OPTICAL FIBERS}

To overcome the issues of low transverse strain sensitivity and significant thermal cross-sensitivity of conventional highly birefringent fibers, we propose to work with highly birefringent microstructured optical fibers (MOF). Our previous research resulted in a dedicated MOFBG sensor ('butterfly MOF', Figure 1) with unprecedented transverse strain sensitivity, combined with a very low thermal cross sensitivity. By aligning the angular orientation of the transverse strain sensing axes of the butterfly MOFBG sensor with the direction of principal stress in a shear loaded material, we have shown previously that this sensor can also be used for shear stress sensing [3].

The sensing principle of the butterfly MOFBG sensor relies on the change of modal birefringence $B_{\text {mod }}$ caused by a mechanical load that is applied to the cladding of the fiber, or to the host material in which the sensor is embedded [4]. Because of the asymmetric microstructure, a radial load will induce an asymmetric mechanical stress distribution in the core of the fiber. The refractive indices $n_{x}$ and $n_{y}$ of the modes polarized along the $x$ - and $y$-direction are therefore affected in a different manner. This is translated into a change of the separation $\Delta \lambda$ between the individual Bragg peak wavelengths $\lambda_{i}$ for each linearly polarized mode, which is directly linked to $B_{\text {mod }}$ as given by $\Delta \lambda=2$. $B_{\bmod } \Lambda$, with $\Lambda$ the period of the FBG. We consider $\Delta \lambda$ as the sensor signal, and the sensitivity of the butterfly MOFBG sensor corresponds to the rate of change of $\Delta \lambda$ when submitted to a specific mechanical or thermal load. The butterfly MOFBG sensor has a sine-like dependence of the transverse strain sensitivity to the angular orientation of the microstructure with respect to the direction of the applied load. It is most sensitive when the load is applied along $\alpha=90^{\circ}$, which is indicated by the slow-axis or $y^{\prime}$-axis in Figure 1 [2]. However, when the sensor is embedded in a shear loaded material at an angular orientation $\alpha=+45^{\circ}$ or $\alpha=-45^{\circ}$, it will detect the transverse strain that is induced by the shear load. By orienting the sensor at $+45^{\circ}$ or $-45^{\circ}$, the fundamental optical axes are aligned parallel to the directions of principal strain in the material. 


\section{SHEAR STRESS SENSING WITH A BUTTERFLY MOFBG SENSOR IN DIFFERENT ADHESIVE BOND CONFIGURATIONS}

We test the shear stress sensitivity of the butterfly MOFBG sensor in various adhesive bond configurations. Figure 2 gives a schematic overview of the different bond types and the locations along the bond overlap at which sensors have been embedded. The instrumented joints are subjected to a static tensile load by gripping them at both ends in a hydraulic servo-controlled tensile test machine with a load capacity of max. $100 \mathrm{kN}$. During loading, the FBG sensor response is recorded using an FBG interrogator (FBG-scan 608 [5]). Linear regression analyses of the responses of the MOFBG sensors to tensile loading $F$ result in sensor sensitivities expressed in $\mathrm{pm} / \mathrm{kN}$. The theoretical model of Goland-Reissner [6] is used to relate the applied load to the shear stress at the position of the sensor, while the low amount of peel stress present at the sensor location is neglected. This allows calculating the shear stress sensitivity, expressed in $\mathrm{pm} / \mathrm{MPa}$. After testing the samples up to failure, their sides were polished in order to determine the exact angular orientation of the embedded optical fibers with a microscope.

Three sensors have been embedded in single lap adhesive joints (SLJ), bonded by different types of adhesive; two types of epoxy-based adhesives (Araldite $420 \mathrm{~A} / \mathrm{B}$ and Araldite 2015) and one methyl methacrylate adhesive (Supergrip MMA 8105). Their material parameters are listed in Table 1, along with the results of their shear stress sensitivities, which are $59.7 \mathrm{pm} / \mathrm{MPa}, 63.3 \mathrm{pm} / \mathrm{MPa}$ and $59.8 \mathrm{pm} / \mathrm{MPa}$ for respectively sample A.1, A.2 and A.3.
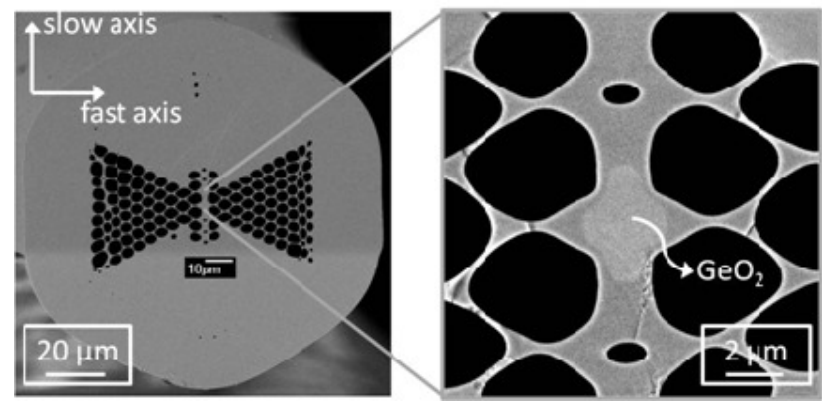

a.

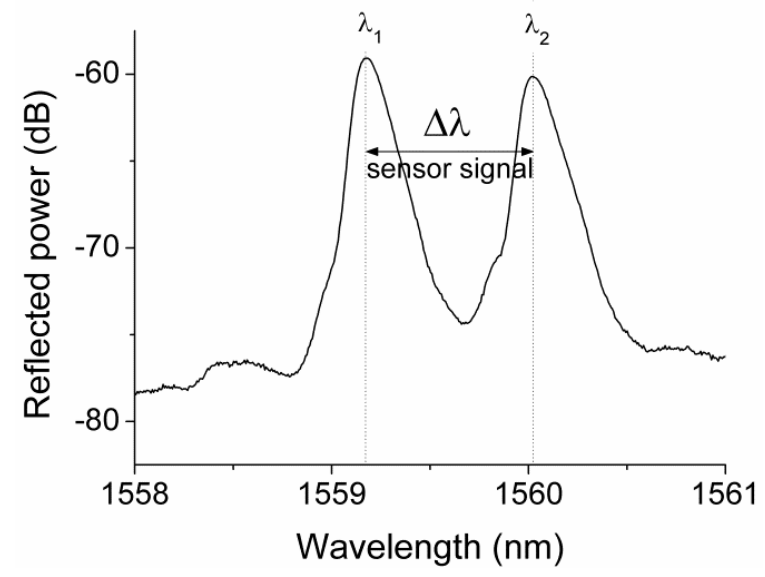

b.

Figure 1: a. Cross sectional view of the 'butterfly' microstructured optical fiber Bragg grating (MOFBG) sensor. The specific asymmetric air hole topology results in a MOFBG sensor with unprecedented shear stress sensitivity. b. Optical reflection spectrum of a butterfly MOFBG sensor. The peak separation $\Delta \lambda$ is used as sensor signal to measure shear loading. 
These values demonstrate that the sensor response does not depend on the type of adhesive in which it is integrated.

\begin{tabular}{|l|c|c|c|}
\hline Sample & Adhesive type & $\begin{array}{c}\text { Sensor } \\
\text { orientation }\end{array}$ & $\begin{array}{c}\text { Shear stress } \\
\text { sensitivity }\end{array}$ \\
\hline A.1 & Araldite $420 \mathrm{~A} / \mathrm{B}(E=2000 \mathrm{MPa}, v=0.35)$ & $-52.1^{\circ}$ & $59.7 \mathrm{pm} / \mathrm{MPa}$ \\
\hline A.2 & Araldite $2015(E=1850 \mathrm{MPa}, v=0.42)$ & $-47.3^{\circ}$ & $63.3 \mathrm{pm} / \mathrm{MPa}$ \\
\hline A.3 & Supergrip MMA $8105(E=470 \mathrm{MPa}, v=0.39)$ & $-39.9^{\circ}$ & $59.8 \mathrm{pm} / \mathrm{MPa}$ \\
\hline
\end{tabular}

Table 1: Influence of type of adhesive on the shear stress sensitivity of butterfly MOFBG sensors embedded in the centre of a single lap adhesive joint. The material parameters (elastic modulus $E$ and Poisson ratio $v$ ) of the adhesive are provided.

Since many different joint configurations are used in practical applications, we also test the shear stress sensitivity of the butterfly MOFBG sensor when integrated in a double lap joint (DLJ). Because of its symmetric configuration, the peel stress level in this bond type is much lower than that in a single lap joint. Hence, we can expect that if the butterfly MOFBG sensor is cross-sensitive to peel and shear stress, the peel stress will only have a limited effect when it is embedded in a DLJ. Table 2 compares the shear stress sensitivities when embedded in a SLJ (59.7 $\mathrm{pm} / \mathrm{MPa})$ and when embedded in a DLJ $(52.9 \mathrm{pm} / \mathrm{MPa})$. We can therefore assume that peel stress has a negligible effect on the butterfly MOFBG shear stress sensor when it is embedded centrally in a SLJ.

\begin{tabular}{|l|c|c|c|}
\hline Sample & Joint configuration & $\begin{array}{c}\text { Sensor } \\
\text { Orientation }\end{array}$ & $\begin{array}{c}\text { Shear stress } \\
\text { sensitivity }\end{array}$ \\
\hline B.1 = A.1 & Single lap & $-52.1^{\circ}$ & $59.7 \mathrm{pm} / \mathrm{MPa}$ \\
\hline B.2 & Double lap & $-53.3^{\circ}$ & $52.9 \mathrm{pm} / \mathrm{MPa}$ \\
\hline
\end{tabular}

Table 2: Influence of joint configuration on the shear stress sensitivity of butterfly MOFBG sensors embedded in the centre of an adhesive joint. The adhesive is two-component epoxy based Araldite 420 A/B.

\section{single lap adhesive joint}
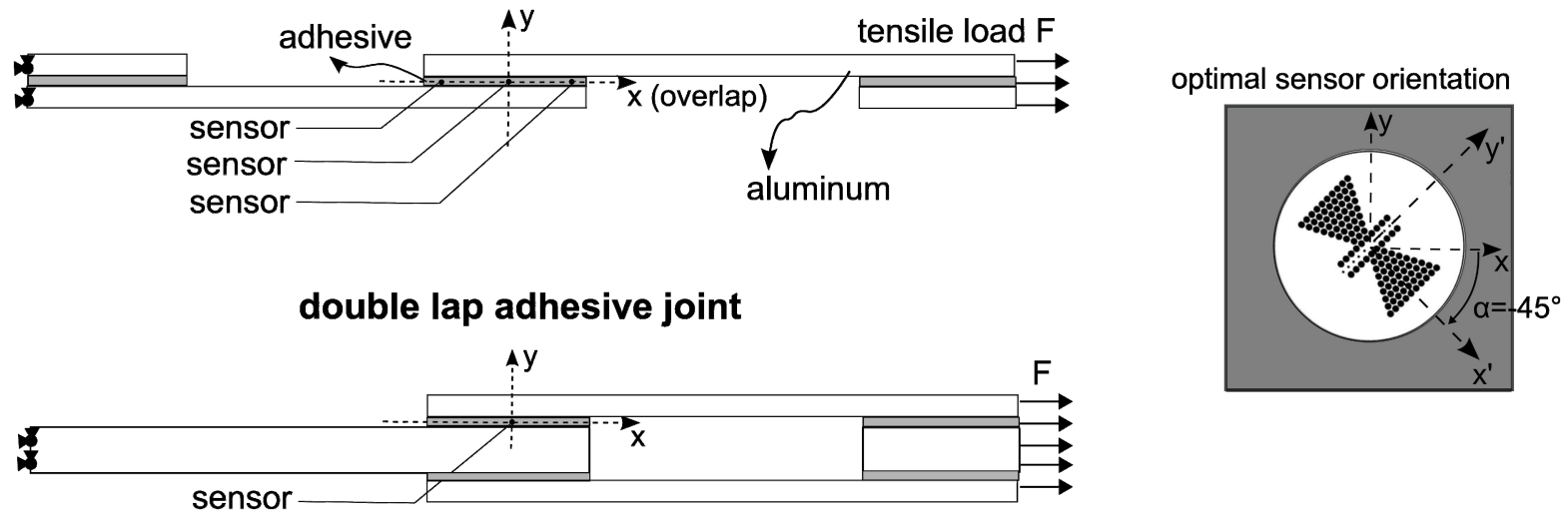

Figure 2: Schematic overview of a single lap and a double lap joint configuration, with the location of the embedded sensors indicated. Besides testing different joint configurations, we have also investigated the sensitivity when different types of adhesives are used. 
The previous results demonstrate that we can monitor the shear stress centrally in an adhesive bond in a repeatable manner for different adhesive types and joint configurations. When the sensors are installed in the adhesive bond for quality monitoring purposes, it can be more advantageous to install them at locations where the shear stress is higher, i.e. closer to the edges of the overlap. However, peel stress also becomes larger near the edges. There are nevertheless two specific locations closer to the edge at which peel stress is nearly zero, while shear stress is higher than in the centre of the adhesive [6]. In our experiments we opted to integrate the sensors at these locations (Figure 2).

In addition to studying the influence of the sensor location on the sensor response, we also investigated the effect of the angular orientation of the sensor. It might be of interest to have sensors for shear stress sensing, which should be oriented at $+45^{\circ}$ or $-45^{\circ}$, and at the same time integrate sensors for transverse stress sensing that need to be

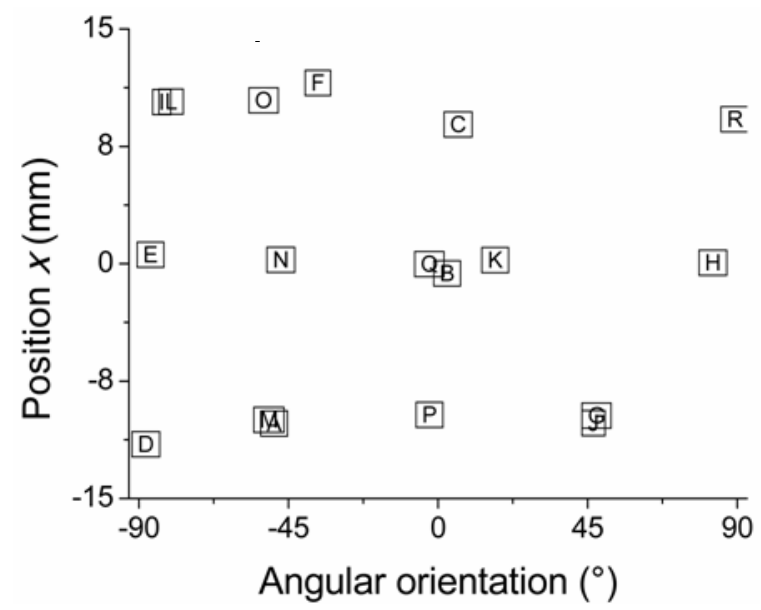

(a.)

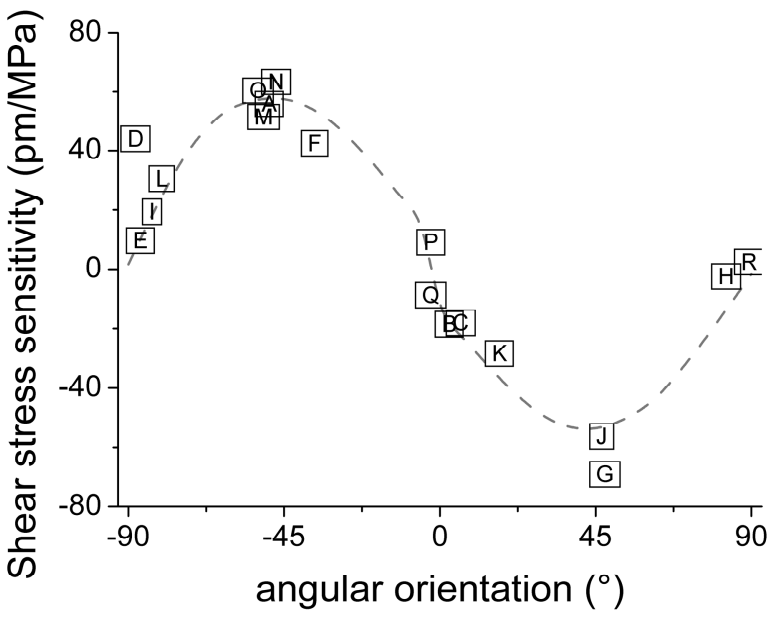

(b.)

Figure 3: A total of $6 \mathrm{SLJ}$ samples, each containing 3 embedded sensors, are tested. The single lap joint is bonded by a two-component epoxy based Araldite 2015 adhesive. The dimensions and embedding details of the sensors are summarized in (a). (b) shows the sine-like angular dependence of the shear stress sensitivity of the sensors. The sensitivity is maximal for an orientation of $\mathbf{- 4 5 ^ { \circ }}$.

embedded preferably at $0^{\circ}$. Figure 3 a gives an overview of the orientations of the tested sensors and at which locations along the overlap length they were embedded.

Figure $3 \mathrm{~b}$ summarizes the results of the shear stress sensitivity of the different sensors. The angular dependence of the shear stress sensitivity follows a sine-like trend. This is in correspondence with the sine-like trend of the transverse strain sensitivity [2]. Moreover, the sensitivity reaches its maximum when the sensor is embedded at an angular orientation of $+45^{\circ}$ and $-45^{\circ}$ (respectively $-69.0 \mathrm{pm} / \mathrm{MPa}$ and $63.3 \mathrm{pm} / \mathrm{MPa}$ ), while at an orientation of $0^{\circ}$ or $90^{\circ}$ the sensitivity decays. These results demonstrate that by changing the angular orientation of the butterfly MOFBG sensor, it can be used for either shear strain sensing or for transverse strain sensing. Moreover, it also shows that the response of the butterfly MOFBG sensor to shear loading is independent of its location along the bond overlap when peel stress is absent. 
Our decision to work with highly birefringent microstructured optical fibers is mainly related to their potential for mechanical stress sensing with a high sensing resolution, while offering possibilities to maintain a low thermal cross sensitivity. We experimentally investigate the thermal cross-sensitivity of the shear strain sensitivity when the sensor is embedded at an angular orientation of $-42^{\circ}$ in the centre of a single lap adhesive joint (Araldite $420 \mathrm{~A} / \mathrm{B}$ ). The tensile loading test is repeated at $20^{\circ} \mathrm{C}$, at $35^{\circ} \mathrm{C}$ and $50^{\circ} \mathrm{C}$, and the shear stress sensitivity is determined at each temperature. A temperature of $50^{\circ} \mathrm{C}$ is at the upper limit of the usable range of the adhesive. We find that the response of the shear stress sensor increases by $8 \%$ when the test is performed at $35^{\circ} \mathrm{C}$, and that in increases again by $30 \%$ when the test is performed at $50^{\circ} \mathrm{C}$. The strong increase in sensor response at $50^{\circ} \mathrm{C}$ can be attributed to the decreased adhesive performance. These results demonstrate that small temperature fluctuations will have a small influence on the shear stress measurements.

\section{CONCLUSION}

We have experimentally verified that butterfly MOFBG based shear stress sensors can be used to monitor the shear stress in the centre of different configurations of adhesive joints bonded by different types of adhesives. The shear stress sensitivity of this type of sensor to shear loading of the bond is about $60 \mathrm{pm} / \mathrm{MPa}$ for these different configurations. We have also shown that shear stress can be measured at different locations along the bond overlap, which opens up possibilities for distributed shear stress mapping. By changing the angular orientation of the butterfly MOFBG sensor it can be used for either shear strain sensing or for transverse strain sensing, which provides opportunities for multi-axial strain sensing with the same type of sensor. Finally, we have demonstrated the minimal thermal cross-sensitivity of the shear stress sensitivity of this sensor. Our results demonstrate the potential of Bragg grating-based sensors for shear stress sensing, and emphasize the added value of dedicated microstructured optical fiber sensors for adhesive bond monitoring.

\section{ACKNOWLEDGEMENT}

The authors would like to acknowledge financial support from the Agency for Innovation by Science and Technology (IWT) for funding this research with doctoral grant (101221) and with the SBO Project SSC grant 120024. The authors also acknowledge the Research Foundation - Flanders (FWO), the Methusalem and Hercules Foundations Flanders, and the COST action TD1001.

\section{REFERENCES}

[1] G. Luyckx, E. Voet, T. Geernaert, K. Chah, T. Nasilowski, W. De Waele, W. Van Paepegem, M. Becker, H. Bartelt, W. Urbanczyk, J. Wojcik, J. Degrieck, F. Berghmans, and H. Thienpont, "Response of FBGs in Microstructured and Bow Tie Fibers Embedded in Laminated Composite," IEEE Photonics Technol. Lett. 21, 1290-1292 (2009).

[2] C. Sonnenfeld, S. Sulejmani, T. Geernaert, S. Eve, N. Lammens, G. Luyckx, E. Voet, J. Degrieck, W. Urbanczyk, P. Mergo, M. Becker, H. Bartelt, F. Berghmans, and H. Thienpont, "Microstructured Optical Fiber Sensors Embedded in a Laminate Composite for Smart Material Applications," Sensors 11, 2566-2579 (2011).

[3] S. Sulejmani, C. Sonnenfeld, T. Geernaert, G. Luyckx, D. Van Hemelrijck, P. Mergo, W. Urbanczyk, K. Chah, C. Caucheteur, P. Megret, H. Thienpont, F. Berghmans, "Shear stress sensing with Bragg grating-based sensors in microstructured optical fibers," Optics Express 21, 20404-20416 (2013).

[4] T. Martynkien, G. Statkiewicz-Barabach, J. Olszewski, J. Wojcik, P. Mergo, T. Geernaert, C. Sonnenfeld, A. Anuszkiewicz, M. K. Szczurowski, K. Tarnowski, M. Makara, K. Skorupski, J. Klimek, K. Poturaj, W. Urbanczyk, T. Nasilowski, F. Berghmans, and H. Thienpont, "Highly birefringent microstructured fibers with enhanced sensitivity to hydrostatic pressure," Opt. Express 18, 15113-15121 (2010).

[5] www.fbgs.com

[6] M. Goland and E. Reissner, J. Appl. Mech. Trans. Am. Soc. Eng., 66, A17 (1944). 